\title{
Les ateliers de l'éthique
}

The Ethics Forum

\section{Penser la question des rapports aux savoirs en éducation : clarification et besoin de recherches conceptuelles}

\section{Mathieu Gagnon}

Volume 6, numéro 1, printemps 2011

URI : https://id.erudit.org/iderudit/1044300ar

DOI : https://doi.org/10.7202/1044300ar

Aller au sommaire du numéro

Éditeur(s)

Centre de recherche en éthique de l’Université de Montréal

ISSN

1718-9977 (numérique)

Découvrir la revue

Citer cet article

Gagnon, M. (2011). Penser la question des rapports aux savoirs en éducation : clarification et besoin de recherches conceptuelles. Les ateliers de l'éthique / The Ethics Forum, 6(1), 30-42. https://doi.org/10.7202/1044300ar

\section{Résumé de l'article}

Ce texte examine la question des rapports aux savoirs par la mise en évidence d'enjeux conceptuels, auxquels se rapportent des enjeux éducatifs et éthiques. À cet égard, l'auteur propose un essai de classification et d'organisation par le recours, notamment, à quatre types de rapports aux savoirs.
Tous droits réservés (C Centre de recherche en éthique de l'Université de Montréal, 2011
Ce document est protégé par la loi sur le droit d'auteur. L'utilisation des services d'Érudit (y compris la reproduction) est assujettie à sa politique d'utilisation que vous pouvez consulter en ligne.

https://apropos.erudit.org/fr/usagers/politique-dutilisation/ 


\section{0}

\section{VOLUME 6 NUMÉRO PRINTEMPS/SPRING 2011}

PENSER LA QUESTION DES RAPPORTS AUX SAVOIRS EN ÉDUCATION : CLARIFICATION ET BESOIN DE RECHERCHES CONCEPTUELLES

MATHIEU GAGNON

UNIVERSITÉ DU QUÉBEC À CHICOUTIMI

\section{RÉSUMÉ}

Ce texte examine la question des rapports aux savoirs par la mise en évidence d'enjeux conceptuels, auxquels se rapportent des enjeux éducatifs et éthiques. À cet égard, l'auteur propose un essai de classification et d'organisation par le recours, notamment, à quatre types de rapports aux savoirs.

\section{ABSTRACT}

This paper focuses on the question of the relationships to knowledge by highlighting conceptual, educational and ethical issues. In this regard, the author proposes an essay of classification and organization by the use, among others, of four types of relationships to knowledge.. 


\section{POUROUOI PENSER LE(S) RAPPORT(S) AU(X) SAVOIR(S) EN ÉDUCATION?}

Bien qu'en éducation l'axe du (des) rapport(s) au(x) savoir(s) (RS) en était un en émergence au début des années 2000, il fait désormais partie du paysage de la recherche et s'impose maintenant comme une dimension fondamentale de la dynamique d'enseignement-apprentissage. De fait, il y a une véritable prolifération des écrits et des études à ce sujet, et ce, tant à l'intérieur du courant francophone que du courant anglo-saxon. Ces écrits, comme nous le verrons, présentent nombre de points de convergence malgré l'absence de dialogue systématique entre la perspective francophone et anglophone. Parmi ces points, il y a un consensus de plus en plus large autour de l'idée que les rapports aux savoirs des élèves et des enseignants, et avec lui la question de l'épistémologie personnelle, seraient des facteurs non négligeables de la persévérance et de la réussite scolaire. Conséquemment, la question de l'épistémologie en éducation, notamment lorsqu'elle est examinée sous l'angle du (des) RS, ne se limite plus à de simples considérations idéologiques ou purement théoriques, mais devient un élément sur lequel tant les didacticiens ${ }^{1}$ et les pédagogues que les enseignants et les élèves (ou étudiants) gagneraient à centrer leur attention. Cet appel à la pensée attentive s'inscrit à l'intérieur d'une perspective résolument transversale. En effet, l'épistémologie se rapporte à toutes disciplines, à tous domaines de formation et donc à toutes matières scolaires. Or, comme nous le verrons (et comme nous l'avons indiqué à l'intérieur d'écrits antérieurs), le caractère transversal de l'épistémologie en éducation doit conduire à considérer les processus de cognition épistémique comme faisant partie intégrante de tous les domaines de formation. Bref, la communauté des chercheurs insiste de plus en plus afin que, d'une part, $i$ $\mathrm{y}$ ait un volet clair de la formation initiale à l'enseignement qui soit consacrée à l'épistémologie en éducation et que, d'autre part, les enseignants invitent leurs élèves à mener régulièrement des réflexions de nature épistémologique.

Nous pourrions également relever des raisons d'ordre éthique afin de fonder l'importance de penser les RS en éducation, dans la mesure où ils teintent en quelque sorte notre rapport au monde. En effet, comme le souligne judicieusement Charlot (1997), être au monde, c'est inévitablement s'inscrire à l'intérieur d'une culture qui ellemême se construit par les relations qu'elle entretient avec les diffé- rents savoirs, lesquels sont portés, notamment, par des institutions. Nos rapports au monde sont filtrés et guidés par des « déjà-là », par des corpus constitués envers lesquels nous entretenons des relations particulières, voire différenciées. Ces relations sont en quelque sorte tributaires de notre société d'attache et elles contribuent à construire notre identité épistémique. L'individu se situe par rapport aux divers corpus de savoirs, leur accorde une certaine valeur, un certain statut et se constitue comme sujet connaissant en développant son «moi épistémique ». L'épistémologie personnelle, souvent implicite, représente en ce sens un enjeu éthique puisqu'elle est liée au rapport que nous avons envers nous-mêmes, à la conception de nos propres capacités et compétences, voire aux manières dont nous nous approprions notre liberté et agissons comme citoyen. Les enjeux éthiques de la question des RS en éducation se rapportent à l'inévitable relation savoir-pouvoir, pour emprunter les mots de Foucault, dans laquelle ils nous plongent. En effet, la posture épistémologique que nous empruntons participe du partage des pouvoirs selon, notamment, la valeur et le statut accordés à l'expertise. Considérer, par exemple, les experts comme des porteurs de vérités objectives auxquelles nous n'avons pas nécessairement accès, c'est leur accorder un pouvoir multiforme pouvant s'exprimer à l'intérieur d'une grande variété de contextes. Conséquemment, il devient précieux d'éveiller les élèves aux dimensions épistémologiques des savoirs qui leur sont présentés en classe, à leur processus d'élaboration et à leur statut afin de favoriser le raffinement de leurs conceptions épistémologiques. Il en va, indirectement, de l'exercice de leur citoyenneté.

Les quelques considérations précédentes ne font voir qu'une partie des enjeux liés à l'épistémologie en éducation. Par contre, la quantité des raisons avancées ne peut servir de critère si elle n'est pas accompagnée d'un espace réflexif permettant de clarifier ce dont nous parlons, d'examiner les différents modèles afin d'en relever des points de convergence et de tension, d'identifier des besoins de recherche et de proposer des pistes à explorer. C'est cette volonté qui anime le présent texte. En ce sens, nous nous demanderons comment sont définies les notions de RS et de croyances épistémologiques, quelles relations elles entretiennent, quels sont les concepts s'y rattachant et de quelles manières ils sont organisés. Partant, nous serons conduits à relever des besoins de recherches conceptuelles pour lesquelles nous proposons des pistes de réflexion. Soulignons cependant qu'il ne s'agit que d'une réflexion embryonnaire dont les avenues suggérées ne doi- 
vent tout au plus être considérées que comme de la " matière à penser ». Mais malgré ce caractère exploratoire, il n'en demeure pas moins que cette matière donne à penser et que, déjà, il est possible d'identifier des besoins de recherche sur une question qui revêt de plus en plus d'importance en éducation.

\section{PENSER LE(S) RAPPORT(S) AU(X) SAVOIR(S) EN ÉDUCA- TION : CLARIFICATIONS ET BESOINS DE RECHERCHES CONCEPTUELLES}

D'entrée de jeu, nous avons indiqué que le (les) RS se situent dans l'axe de l'épistémologie en éducation. Or, d'un point de vue proprement étymologique, le terme épistémologie signifie un discours rationnel (logos : discours; raison) sur les savoirs et/ou les connaissances (epistêmê : connaissances "scientifiques »; savoirs). Ainsi, lorsque nous parlons de la cognition épistémique, nous faisons référence aux processus de pensée portant sur le niveau de certitude des connaissances, leurs limites, leurs critères ainsi que sur les stratégies permettant de les construire ou de résoudre des problèmes (Kitchener, 1983). Dès lors, dans la mesure où le(s) RS est défini comme " une relation de sens, et donc de valeur, entre un individu (ou un groupe) et les processus ou produits des savoirs » (Charlot, Bautier et Rochex, 1992, p.29; Charlot, 2003), nous pouvons poser que ce(s) rapport(s) touche l'épistémologie, car toute entreprise de construction de sens et de réflexivité à l'égard des processus et produits des savoirs constitue en quelque sur un discours rationnel (logos) sur les connaissances, les savoirs (epistêmê). Cependant, bien que foncièrement épistémologique, les questions relatives au(x) RS ont été traitées selon des perspectives différentes qui chacune conduit à les examiner et à les comprendre d'une manière particulière, en dirigeant l'attention vers des composantes distinctes.

\section{REGARDS SUR LES COURANTS FRANCOPHONE ET ANGLO-SAXON}

Comme le souligne Therriault (2008), au moins trois perspectives émergent du courant francophone, à savoir 1) psychanalytique; 2) sociologique et 3 ) didactique. La perspective psychanalytique, telle que développée notamment par Beillerot (1989) et Mosconi et al. (2000), examine le savoir comme objet de désir et concerne, au cœur de la dynamique d'apprentissage, le désir d'apprendre et de connaître. La perspective sociologique quant à elle, développée notamment par Charlot (1997, 2003) ainsi que Charlot, Bautier et Rochex (1992), contribua à jeter les bases des considérations didactiques relatives $\mathrm{au}(\mathrm{x}) \mathrm{RS}$. À l'origine, cette perspective visait à redéfinir l'échec scolaire en insistant, notamment, sur le fait que la scolarité est avant tout une histoire singulière qui dépasse en quelque sorte les dichotomies " favorisé-défavorisé ». Dans ce contexte, le(s) RS est d'abord considéré comme un rapport au monde: le savoir constitue un " déjà-là », souvent incarné par des institutions auxquelles se rattachent des paradigmes spécifiques et qu'il s'agit de s'approprier. Dès lors, le(s) RS se définit comme le sens créé par l'individu qui, inévitablement, est confronté à la nécessité d'apprendre. Cette nécessité d'apprendre participe d'une histoire particulière certes, mais elle participe également d'un contexte social et culturel qui l'oriente en privilégiant (sur la base de critères variés tels la tradition, l'utilité, la valeur scientifique, etc.) certains corpus plutôt que d'autres. Confronté ainsi à la nécessité naturelle et sociale d'apprendre des savoirs portés par des institutions, dont l'école, le(s) RS est (sont) envisagé(s) plus particulièrement sous l'angle du rapport à l'apprendre, c'est-à-dire du rapport à soi comme sujet connaissant, du rapport à l'école, à la classe et à l'enseignant (contrat didactique). La perspective didactique quant à elle (Albe et Venturini, 2002; Caillot, 2001; Jonnaert et Vander Borght, 1999; Larochelle et Désautel, 2004; Jonnaert et Lenoir, 1993; Maury et Caillot, 2003; Therriault, 2008; Venturini, 2007), dirige davantage l'attention sur le(s) RS des étudiants (principalement de niveau collégial et universitaire) et des enseignants, sur les relations didactiques entre l'étudiant et/ou l'enseignant et la matière, de même que sur les rapports aux savoirs scientifiques par le biais de concepts spécifiques.

A l'intérieur du courant anglo-saxon, la question du (des) RS a été développée principalement dans le champ de la psychologie cognitive autour du concept plus spécifique de «croyances épistémologiques» (epistemological beliefs). De manière générale, la plupart des études menées dans ce courant tente de déterminer, par le biais de questionnaires, l'épistémologie personnelle des répondants et de dessiner des relations entre cette épistémologie et différents facteurs, dont la culture, la réussite et la persévérance scolaire, etc. Actuellement, il semble se dégager un certain consensus parmi les chercheurs sur la manière d'opérationnaliser le concept de croyances épistémologiques, à savoir selon deux axes, l'un touchant la nature du savoir, l'autre l'acte de connaître, chacun étant composé de deux continuums. Voici de quelle manière pourrait être illustrées ces composantes: 


\section{A- Nature du savoir}

Les savoirs sont (correspondent à)...

Une série de faits isolés — Des réseaux complexes de concepts

Fixes

Un processus en évolution

B- Acte de connaître

Notre capacité d'apprendre est...

Innée

En développement

L'apprentissage...

S'effectue rapidement ou pas du tout Correspond à un processus graduel demandant investissement et efforts

Ces axes et continuums ont été élaborés sur la base de différents modèles épistémologiques développementaux considérés fondateurs dans le domaine. Parmi ceux-ci, nous retrouvons les travaux de Perry (1970), de Belenky et al. (1896), de Baxter Magolda (2002), de King et Kitchener $(1994,2002)$ de même que de Kuhn (1991). Ces modèles lyse de données empiriques et qu'ils n'ont pas été construits a priori. Chacun de ces modèles propose des stades à travers lesquels les adultes (jeunes et moins jeunes) passent afin de raffiner leurs conceptions épistémologiques. Cependant, bien qu'il soit possible de relever des points de recoupement entre les différents stades identifiés, ceuxci ne renvoient pas toujours aux mêmes conceptions ni aux mêmes perspectives. Plusieurs raisons permettent d'expliquer cette situation, parmi lesquelles se retrouvent les échantillonnages ainsi que les visées poursuivies et les contextes des études desquelles ont émergé ces modèles. À cet égard, le tableau proposé par Hofer et Pintrich en 1997 offre une excellente synthèse des étapes identifiées ainsi que des perspectives empruntées. Reprenons ce tableau en y apportant quelques modifications, notamment en y intégrant un modèle plus récent, à savoir celui de Daniel et al. (2004)

Tableau 1 (inspiré de Hofer et Pintrich, 1997)

sont considérés émergents dans la mesure où ils sont issus de l'ana-

Modèles du développement épistémologique

\begin{tabular}{|l|l|l|l|l|l|}
\hline $\begin{array}{l}\text { Développement } \\
\text { intellectuel et } \\
\text { éthique (Perry) }\end{array}$ & $\begin{array}{l}\text { Manières d'appren- } \\
\text { dre ches les femmes } \\
\text { (Belensky et al. 1986) }\end{array}$ & $\begin{array}{l}\text { Réflexion } \\
\text { épistémologique } \\
\text { (Baxter Magolda, } \\
\text { 2002) }\end{array}$ & $\begin{array}{l}\text { Cognition } \\
\text { épistémique (King } \\
\text { et Kitchener, 2002) }\end{array}$ & $\begin{array}{l}\text { Raisonnnement } \\
\text { argumentatif } \\
\text { (Kuhn, 1990) }\end{array}$ & $\begin{array}{l}\text { Pensée critique } \\
\text { dialogique } \\
\text { (Daniel et al, 2004) }\end{array}$ \\
\hline Positions & $\begin{array}{l}\text { Perspectives } \\
\text { épistémologiques }\end{array}$ & Statut des savoirs & $\begin{array}{l}\text { Cognition } \\
\text { épistémique }\end{array}$ & $\begin{array}{l}\text { Conceptions } \\
\text { épistémologiques }\end{array}$ & $\begin{array}{l}\text { Perspectives } \\
\text { épistémologiques }\end{array}$ \\
\hline Dualisme & Silence & Savoir Absolu & Réalisme & Réalisme & Égocentrisme \\
\hline Multiplicité & Savoir Reçu & Savoir transitoire & Dogmatisme & Absolutisme & Relativisme \\
\hline Relativisme & Savoir subjectif & Savoir Indépendant & Scepticisme & Multiplicisme & intersujectivité \\
\hline Relativisme engagé & Savoir procédural & Savoir indépendant & Subjectivisme & Évaluation & \\
\hline & Savoir construit & Savoir contextuel & $\begin{array}{l}\text { Relativisme } \\
\text { procédural } \\
\text { Relativisme Global }\end{array}$ & & \\
\hline & & & & & \\
\end{tabular}


À ces différents modèles pourrait s'ajouter celui de Lafortune et al. (2003), construit non pas à partir d'étapes, mais autour d'un continuum allant d'un rapport dogmatique à un rapport relatif au(x) savoir(s). À l'intérieur de ce modèle, les critères liés au rapport dogmatique sont la certitude, l'irréfutabilité, l'a-temporalité, la conservation, la généralité et l'universalité, alors que ceux constituant le rapport relatif au(x) savoir(s) sont plutôt l'incertitude, la réfutabilité, la temporalité, le changement, la spécificité et l'individualité. Nous pourrions également faire appel à la perspective de Jonnaert et Vander Borght (1999) articulée autour du concept de posture épistémologique, c'est-à-dire le « cadre général ou le paradigme épistémologique auquel se réfère l'enseignant [nous pourrions dire l'individu] lorsqu'il est question d'acquisition, de développement ou de construction de connaissances »(Therriault, 2008). Bien que la perspective de Jonnaert et Vander Borght soit d'abord didactique, elle conduit tout de même à relever que les postures épistémologiques ancrent le(s) RS et participent de différents courants. À cet égard, Jonnaert et Vander Borght (1999) retiennent trois paradigmes principaux, à savoir l'empirisme, l'idéalisme et le rationalisme. Selon eux, " différents paradigmes épistémologiques se distribuent [...] à travers ces trois courants » $(1999$, p. 27).

\section{VERS UNE SYNTHĖSE DES COURANTS : REGARDS SUR \\ LA MULTIPLICITÉ}

Ces quelques clarifications témoignent de la variété des modèles en lien avec le(s) RS, notamment sous l'angle des perspectives épistémologiques. Cependant, bien que ces modèles se recoupent par le sens téléologique qu'ils partagent, ils conduisent à jongler avec une multiplicité de notions qui ne sont pas nécessairement équivalentes. Cette multiplicité n'est pas toujours apparente à l'intérieur des modèles développementaux, mais dès l'instant où nous tentons d'en comprendre la signification et l'organisation, nous sommes reconduits vers d'autres notions épistémologiques qui témoignent d'un rapport différent au savoir. Il est dès lors question d'objectivité, d'objectivation, de viabilité, de vérité, de découverte... A cet égard, l'angle retenu semble avoir une incidence sur le type de notions utilisées. C'est pourquoi, par exemple, Belenky et al. (1986) parlent en termes de «savoir reçu», alors que l'équivalent s'exprime chez King et Kitchener (2002) par le recours au concept de dogmatisme. Dans ce cas, il est possible de percevoir des relations intimes entre entretenir des rapports dogmatiques aux savoirs et le fait de s'inscrire, lorsqu'il est question de l'acte d'apprendre, à l'intérieur d'une dynamique de savoir reçu. Seulement, bien que liées, il ne semble pas que ces notions réfèrent à un RS qui soit entièrement du même ordre. Dès lors, il devient pertinent de se questionner sur l'équivalence des différents RS qui semblent se dégager des notions constitutives des modèles, et sur la pertinence de parler du RS en termes de rapport au singulier. À cela s'ajoute le fait qu'un regard porté sur la littérature en épistémologie montre que plusieurs modèles ne permettent pas de rendre compte de certaines théories jugées pourtant charnières, dont celles développées par Karl Popper et Thomas Kuhn. Il s'agit là de l'une des limites inhérentes aux modèles émergents : des données, nous ne pouvons faire ressortir des catégories se situant à l'extérieur de nos cadres (qu'ils soient implicites ou explicites), tout comme le fait de ne pas avoir relevé des catégories n'implique pas nécessairement que celles-ci doivent être exclues de nos démarches d'analyses et de recherches. Or, lorsque nous entreprenons de dresser une liste, non exhaustive de surcroît, des différentes notions avec lesquelles nous sommes en contact lorsque nous examinons à la fois les modèles développementaux et les théories épistémologiques dessinées dans le champ de la philosophie, nous prenons rapidement conscience de leur large éventail. Voici un aperçu des notions qui pourraient faire partie de cette liste ${ }^{2}$ :

1 Vérité

2 Intersubjectivisme / Intersubjectivité

3 Réalisme

4 Dualisme

5 Certitude

6 Apriorisme

7 Relativisme

8 Généralité

9 Changement

10 Relativisme procédural

11 Pragmatisme

12 Incertitude

13 Empirisme logique

14 Dualisme

15 Cartésianisme

16 Behaviorisme 
17 Relativisme global

18 Savoir procédural

19 Universalité

20 Viabilité

21 Dialectique

22 Rationalisme

23 Relationnisme

24 Coélaboration

25 Absolutisme

26 Individualité

27 Savoir contextuel

28 Logique

29 A-logique

30 Découverte

31 Empirisme

32 Objectivité

33 Cognitivisme

34 Fonctionnalisme

35 Instrumentalisme

36 Subjectivisme

37 Égocentrisme

38 Constructivisme

39 Idéalisme

40 Révolutionarisme

41 Matérialisme

42 Temporalité

43 Invention

44 Naturalisme

45 Anarchisme

46 Savoir reçu

47 Évaluation

48 Relativisme engagé

49 A-temporalité

50 Falsificationnisme

51 Scepticisme

52 Multiplicisme

53 Connexionnisme

54 Socioconstructivisme

55 Nominalisme

56 Sensualisme
57 Fonctionnalisme

58 Dogmatisme

59 Conservation

60 Irréfutabilité

61 Positivisme

62 Objectivation

63 Innéisme

64 Spécificité

65 Réfutabilité

VERS UNE SYNTHĖSE DES COURANTS : REGARDS SUR DEUX MODES POSSIBLES D'ORGANISATION

Cette brève exploration nous reconduit d'emblée face à la quantité de notions de même qu'à la complexité qu'appelle tout processus d'organisation. En effet, chacun des termes identifiés présuppose une série de considérations dont certaines s'inscrivent à l'intérieur de paradigmes et renvoient à des théories épistémologiques elles-mêmes complexes qui se déclinent en plusieurs modes. C'est le cas, par exemple, du positivisme qui, suivant les travaux d'Auguste Compte, s'est développé à travers différents courants, dont le positivisme logique du Cercle de Vienne qui se distingue du courant initial structuré autour du positivisme scientifique. Cette diversité, combinée à la dynamique complexe à laquelle elle nous conduit, pose un besoin de recherche conceptuelle : il apparait désormais précieux d'entreprendre des réflexions autour de la possibilité de proposer des organisations par catégories, ce qui permettrait du coup de consolider l'information en fonction de critères distincts et complémentaires. À cet égard, nous pourrions imaginer différents modes d'organisation. Dans ce cas, les intentions et projets poursuivis auront un rôle à jouer quant au choix du ou des modes, notamment par l'identification de catégories. Cette tâche apparaît fondamentale compte tenu du nombre de notions convoquées dans l'axe de l'épistémologie en éducation et du (des) RS. Un tel processus pourrait contribuer à clarifier les angles et les relations, tout en fournissant aux acteurs des cadres à partir desquels ils pourront s'approprier, voire analyser, l'information.

Dans ce contexte, nos réflexions ont conduit à élaborer deux modes possibles d'organisation, lesquels sont considérés complémentaires. Bien entendu, compte tenu de l'état d'avancement des différents travaux quant à l'importance d'organiser l'information, les avenues proposées ne représentent pas davantage que des hypothèses à examiner 
et à évaluer, et non l'aboutissement d'un processus de stabilisation conceptuelle. Il s'agit donc de présenter des pistes afin de contribuer à alimenter les recherches, des pistes qui pour l'heure demeurent dans l'ordre de l'exploration.

\section{RAPPORT AU SAVOIR, RAPPORTS AU SAVOIR, RAPPORT AUX SAVOIRS OU RAPPORTS AUX SAVOIRS ?}

Dans un premier temps, lorsque nous prenons connaissances des différents écrits au sujet du (des) RS nous relevons, principalement lorsque nous tentons d'effectuer des arrimages entre la perspective francophone et anglo-saxonne, que si certains, dont Charlot, parlent de RS au singulier, d'autres parlent de rapports (au pluriel) au savoir (au singulier) (Jonnaert et Vander Borght, 1999), alors que d'autres préfèrent parler de rapports (au pluriel) aux savoirs (au pluriel) (Gagnon, à paraître). L'une des raisons expliquant ces appellations diverses est liée au contexte et à la perspective dans laquelle s'inscrivent les diverses études. Ainsi, par exemple, le fait que Jonnaert et Vander Borght s'inscrivent à l'intérieur d'une perspective didactique les conduit à parler d'une diversités de rapports à un savoir, puisqu'ils voient ces rapports comme étant «dans la tête» de l'élève, et ce rapport est toujours pensé en lien avec un corpus de savoir défini par la situation didactique. Dès lors, puisqu'il y a plusieurs élèves, ceux-ci auront des rapports différents au savoir abordé à l'intérieur de la situation, un savoir qui par ailleurs a subi une transposition didactique.

En outre, ces différentes appellations conduisent à des postures variées. En effet, comme nous le verrons lorsque nous aborderons les recherches menées entre autres dans le domaine de l'épistémologie personnelle, partir de l'idée que le savoir est un tout cohérent conduit à examiner ce rapport de manière décontextualisée, générique et transversale. Inversement, une conception selon laquelle il pourrait exister différents rapports en fonction de savoirs diversifiés conduit à engager des analyses selon une approche davantage située et contextuelle. Ceci étant, à l'intérieur des écrits sur le(s) RS, nous pouvons relever qu'il est effectivement question, selon les auteurs, de différents types de rapports; c'est pourquoi nous privilégions le pluriel au singulier en cette matière. Ces types de rapports au(x) savoir(s) pourraient servir de pierre d'assise à un processus de classification à partir duquel il serait désormais possible de les distinguer dans leur «nature» et leur orientation. Examinons de plus près de quelle manière pourrait être articulée cette organisation.

\section{LA MULTIPLICITÉ DES RAPPORTS COMME PRINCIPE DE CLASSIFICATION}

Comme nous le disions à l'instant, il est possible de relever dans les écrits une multiplicité de rapports à partir de laquelle nous pourrions entreprendre un essai de classification. Parmi ces rapports, il y a d'abord ce que nous pourrions qualifier de rapport épistémique. Par " rapport épistémique », nous entendons la valeur accordée aux produits du savoir, ce qui rejoint à la fois l'aspect " relation de valeur » énoncé par Charlot et al. (1992), et nombre de concepts visant à déterminer le statut des savoirs (relevés notamment par Lafortune et $a l$.), tels que la vérité, la viabilité, la certitude, l'objectivité... Ce type de rapport se traduit par des jugements de valeur à partir desquels nous nous prononçons sur le degré de validité des savoirs, comme c'est le cas par exemple lorsque nous disons des savoirs scientifiques qu'ils sont objectifs. En lien avec ce rapport épistémique ainsi qu'avec la position de Jonnaert et Vander Borght, de Therriault, de Hofer et de Schommer selon qui les rapports au savoir s'appuient sur des postures épistémologiques (souvent implicites), nous pourrions identifier un autre type de rapport que nous qualifions de rapport épistémologique. Alors que le rapport épistémique concernait la valeur accordée, par les individus (voire les sociétés) aux savoirs, le rapport épistémologique quant à lui porte davantage sur les processus d'élaboration et de construction des savoirs, notamment des savoirs savants et scientifiques. Ainsi, lorsque nous envisageons les processus, méthodes et/ou stratégies à partir desquelles les savoirs sont développés par les communautés ou à l'intérieur d'un paradigme défini, nous qualifions ce rapport de " rapport épistémologique ». En ce sens, le rapport épistémologique rejoint l'idée de posture épistémologique, et cette posture peut être considérée comme étant liée de manière intime au rapport épistémique; la posture épistémologique « déterminant 》 en quelque sorte la valeur accordée aux savoirs découlant ou émergent de leurs processus d'élaboration. Partant, nous pourrions ranger, sous la catégorie du rapport épistémologique, les théories de la connaissance élaborées en épistémologie, dont le falsificationisme, le positivisme, le rationalisme cartésien, le socioconstructivisme...

Nous pourrions également identifier d'autres types de rapports aux savoirs touchant, cette fois, aux dynamiques d'apprentissage et de construction de connaissances, selon une perspective plus proprement individuelle. À cet égard, nous pourrions dégager au moins trois types 
distincts de rapports aux savoirs, que nous avons nommés : 1) le rapport didactique (c.-à-d. à l'apprendre); 2) le rapport gnoséologique 3) le rapport idiosyncratique. D'abord, par rapport didactique (à l'apprendre), nous référons au métier et rôle des élèves dans l'acte de s'approprier des savoirs standardisés, lesquels ont été transposés dans une visée didactique (Jonnaert et Vander Borght, 1999). À l'intérieur de cette catégorie, nous pourrions retrouver des notions telles que, par exemple, l'innéisme, la douance, la mémorisation, les capacités... De sorte que le rapport didactique se rattache plus spécifiquement aux conceptions que l'individu ou un groupe d'individus partage quant aux démarches d'apprentissage ainsi qu'à la manière dont ils se conçoivent la dynamique d'apprentissage, notamment en contexte scolaire. Le rapport gnoséologique quant à lui touche plus spécifiquement à la question de la nature et des processus de développement des connaissances. De sorte que cette catégorie regroupe les différentes théories ou conceptions touchant la question du développement cognitifs, dont le constructivisme piagétien, le socioconstructivisme de Vygotski, le cognitivisme, le béhaviorisme... Finalement, nous avons relevé un autre type de rapport, que nous avons qualifié de

rapport idiosyncratique. Ce rapport serait lié aux perspectives à partir desquelles sont abordées les savoirs et les sources de savoirs. Cette catégorie rejoint certains des éléments dégagés par Belenky et al. (1986), de même que les notions touchant la manière d'aborder et de concevoir les savoirs en général. Il s'agit en quelque sorte d'une attitude, d'une conception générale à partir de laquelle l'individu aborde le savoir. Ainsi, nous pourrions regrouper sous cette catégorie des notions ou perspectives telles que le dualisme, le multiplicisme, le relativisme absolu, le scepticisme, le relativisme engagé...

Voici de quelle manière pourraient être présentés, sous forme de tableau, les différents types de rapports que nous avons identifiés et qui constituent, par le fait même, des catégories à partir desquelles nous pourrions organiser et regrouper les différentes notions, concepts et théories générales liés aux rapports au(x) savoir(s) ainsi qu'à l'épistémologie en éducation. Bien entendu, comme nous le soulignions d'entrée de jeu, il s'agit d'un modèle demeurant à ce jour embryonnaire et émergent qui gagnerait à être examiné avec attention, critiqué et modifié le cas échéant.

Tableau 2 : Types de rapports au(x) savoir(s)

\begin{tabular}{|c|c|c|c|c|}
\hline RAPPORT ÉPISTÉMOLOGIQUE & RAPPORT ÉPISTÉMIOUUE & $\begin{array}{l}\text { RAPPORT DIDACTIOUE } \\
\text { (À L'APPRENDRE) }\end{array}$ & RAPPORT GNOSÉOLOGIOUE & RAPPORT IDIOSYNCRATIOUE \\
\hline $\begin{array}{l}\text { - Falsificationnisme } \\
\text { - Positivisme } \\
\text { - Révolutionnarisme } \\
\text { - Pragmatisme } \\
\text { - Socioconstructivisme } \\
\text { - Réalisme } \\
\text { - ... }\end{array}$ & $\begin{array}{l}\text { - A-logique } \\
\text { - A-temporel } \\
\text { - Certitude } \\
\text { - Changement } \\
\text { - Conservation } \\
\text { - Généralité } \\
\text { - Incertitude } \\
\text { - Subjectivité } \\
\text { - Vérité } \\
\text { - Objectivité } \\
\text { - Logique } \\
\text { - Viabilité . ... }\end{array}$ & $\begin{array}{l}\text { - Innéisme } \\
\text { - Maïeutique } \\
\text { - Douance } \\
\text { - Mémorisation } \\
\text { - Capacités en développement } \\
\text { - Schèmes } \\
\text { - Rapidité } \\
\text { - Complexité } \\
\text { - Investissement et effort } \\
\text { - Récepteur } \\
\text { - Constructeur... }\end{array}$ & $\begin{array}{l}\text { - Constructivisme (Piaget) } \\
\text { - Rationalisme } \\
\text { - Constructivisme social } \\
\text { (Vygotski) } \\
\text { - Cognitivisme } \\
\text { - Empirico-réalisme } \\
\text { - Béhaviorisme } \\
\text { - ... }\end{array}$ & $\begin{array}{l}\text { - Dogmatisme } \\
\text { - Dualisme } \\
\text { - Évaluatif } \\
\text { - Relativisme absolu } \\
\text { - Scepticisme } \\
\text { - Relativisme engane } \\
\text { - Relativisme procédural } \\
\text { - Savoir reçu } \\
\text { - ... }\end{array}$ \\
\hline
\end{tabular}




\section{REGARDS SUR LES PORTÉES ET LIMITES DE LA CATÉGORISATION}

Il va sans dire que cet essai de classification constitue une construction, et qu'en ce sens, il ne permet pas de ranger chacun des concepts sous une catégorie toute désignée. Il est parfois des éléments qui ne peuvent être réduits à l'une ou l'autre de celles-ci de manière spécifique et exclusive. Cela est tout à fait normal par ailleurs, puisque le monde, qu'il soit question d'une réalité physique ou plus proprement conceptuelle, ne se réduit jamais à un amalgame de catégories, tant et si bien que parfois, un item se rapporte à plusieurs d'entre elles. L'organisation, et avec elle les processus de catégorisation et de classification qui l'accompagnent, sont des outils de pensée qui visent à contribuer au développement de notre compréhension d'un phénomène ou d'une réalité complexe. La présentation de ce tableau s'inscrit donc dans une volonté de contribuer aux processus visant à structurer, autant que faire se peut, notre compréhension de la diversité des rapports aux savoirs manifestés à l'intérieur des écrits. Ainsi, par l'identification de quatre types de rapports se définissant notamment par des critères distincts, nous souhaitons proposer un cadre à l'intérieur duquel pourra être regroupée une série de concepts selon leurs orientations spécifiques et les perspectives dans lesquelles ils s'inscrivent.

\section{REGARDS SUR UNE ORGANISATION EN AXES CROISÉS}

Par ailleurs, nous pourrions également imaginer une organisation des différentes conceptions épistémologiques (i.e. rapports épistémique et/ou épistémologique) non pas cette fois en recourant à des catégories visant à classifier, mais plutôt en s'appuyant sur des couples de critères à partir desquels il serait possible de créer deux axes croisés. En fait, plusieurs modèles ou théories s'articulent autour de la portée épistémique, centrée principalement sur la question de la valeur des savoirs. Ainsi, selon les courants de pensée, certains conduisent à relever que les savoirs sont subjectifs, alors que d'autres tentent d'établir les paramètres permettant d'atteindre l'objectivité exempte de toute forme de subjectivité. D'ailleurs, la question de l'objectivité des savoirs est au cœur de l'épistémologie et des sciences : la première visant, notamment, à déterminer par quels outils il est possible de l'atteindre, les secondes mettant en œuvre des processus, voire des procédures qui permettraient de garantir la valeur de vérité et l'objectivité des savoirs en découlant. À l'opposé, se retrouvent les épistémologies d'inspiration socioconstructiviste qui rejettent la possibilité que les être humains puissent prétendre découvrir la vérité sur les choses, la certitude absolue ou l'objectivité dégagée de toute forme de subjectivité. Cette posture trouve notamment ses origines dans la kantisme où les catégories de l'esprit sont considérées adéquates pour comprendre le monde, mais ne permettent pas pour autant d'atteindre la «réalité nouménale»; nous sommes condamnés, par nature, à nous en remettre à la seule connaissance des phénomènes, et non des choses en elles-mêmes. Cette position trouve également écho dans le texte de Nietzche, Vérité et mensonge au sens extra-moral, un texte dans lequel il exprime que les représentations, ou encore les processus de conceptualisation et de formalisation, sont par nature distants des choses telles qu'elles sont. Cette distance est marquée par deux processus de métaphorisation par lesquels nous parvenons à construire une "réalité rationnelle ». La première métaphorisation consiste en la traduction que notre esprit fait, partant de l'expérience d'une chose, en image de cette chose. Cette image, qui constitue déjà une forme d'abstraction, représente la pierre d'assise d'un processus de rationalisation (seconde métaphorisation) par lequel cette image abstraite est traduite en concept. Ainsi, pour Nietzsche, notre compréhension rationnelle et formelle du monde ne peut constituer la vérité sur les choses. Glasersfeld et Fourez expriment cette idée d'une manière différente en qualifiant 1'objectivité et la prétention à la vérité comme autant de mythes qu'il conviendrait de remplacer par le concept de viabilité. Ainsi, à l'intérieur du constructivisme radical, les savoirs et les connaissances ne sont ni objectifs, ni vrais absolument, ni totalement subjectifs, mais bien viables, c'est-à-dire adéquats et efficaces selon les contextes et projets poursuivis. Partant, les modèles ne sont pas des copies de la réalité telle qu'elle est, mais doivent être considérés comme des constructions détenant un certain pouvoir explicatif et permettant d'obtenir des résultats intéressants selon nos visées.

À la lumière de ces quelques éléments, par trop sommaires il va sans dire, nous pouvons relever qu'il existe des points de tension épistémologique touchant la nature et la valeur des savoirs. Dès lors, nous croyons qu'il pourrait être intéressant d'examiner la portée d'une structure constituée autour de deux axes croisés, lesquels présenteraient des pôles antagonistes qui, par leur croisement, permettraient de situer les différents courants quant aux rapports épistémiques auxquels ils conduisent. Afin de structurer ce modèle en axes croisés, nous avons eu recours à deux couples de critères, couples sur la base 
desquels sont construits nos continuums. Dans un premier temps, nous avons identifié un continuum allant de la subjectivité à l'objectivité, et dans un second temps, nous prenons appui sur un continuum allant de la vérité à la viabilité. Par le croisement de ces axes, nous pensons qu'il pourrait être possible d'organiser les courants épistémologiques de manière à voir où ils se situent sur le plan de la valeur attribuée aux savoirs, notamment des savoirs paradigmatiques. Voici de quelle manière pourrait prendre forme une telle structure en axes épistémiques croisés.

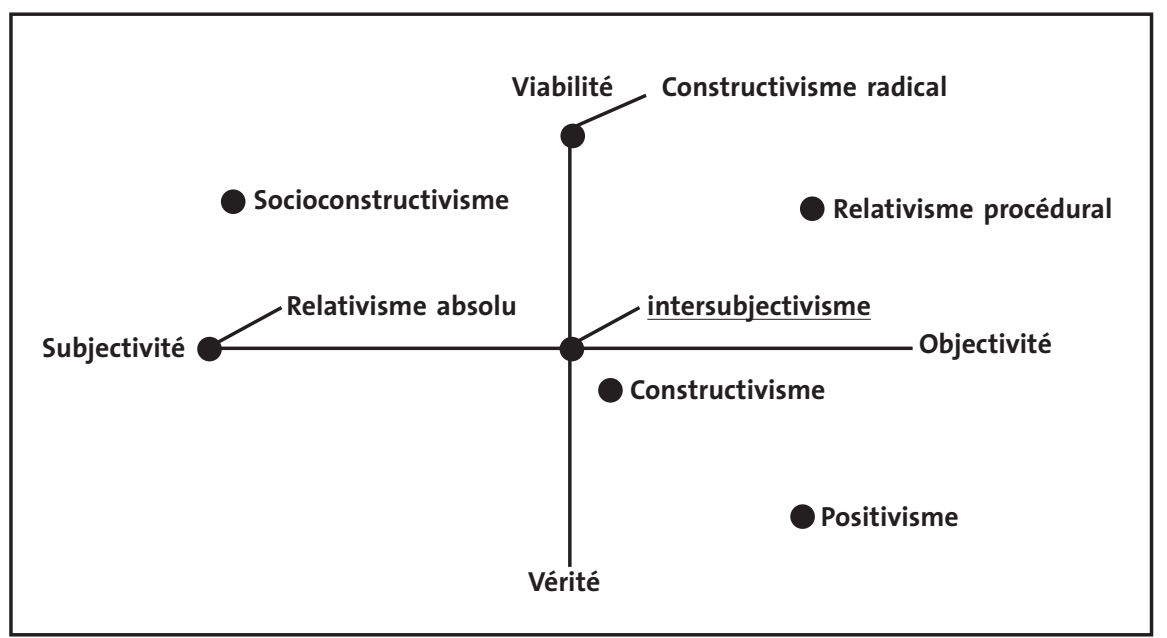

\section{REGARDS SUR LES PORTÉES ET LIMITES D'UNE \\ ORGANISATION EN AXES CROISÉS}

Bien qu'il ne soit pas possible de marquer avec précision l'emplacement des différents courants à l'intérieur d'une telle structure, elle présente tout de même l'avantage (malgré que nous n'ayons inséré que quelques exemples de courants à l'intérieur de la précédente figure) d'offrir un portrait permettant de repérer, par une forme de synthèse, les conceptions épistémiques qui se dégagent des différents courants meublant l'épistémologie. Ainsi, par la situation qu'ils occupent dans cet espace, il serait possible de déterminer plus avant quel courant s'inscrit, par exemple, à l'intérieur d'une perspective selon laquelle les processus ou démarches d'élaboration des savoirs conduisent à découvrir la " vérité ", et laquelle considère qu'au mieux ces processus mènent à la construction de représentations et / ou de modèles viables. Bien entendu, il est possible de relever différentes branches à l'intérieur de ces courants et nous ne pouvons insérer aucun d'entre eux à l'intérieur d'un bloc monolithique sans générer une forme ou une autre de réductionnisme. Ainsi, par exemple, alors qu'il pourrait être entendu que l'intersubjectivisme conduit à l'objectivité, d'autres, dont les socioconstructivistes tels Fourez, indiqueraient que l'intersubjectivité conduit, au mieux, à une forme ou une autre d'objectivation. Néanmoins, un tel modèle, moyennant nuances et précisions, permettrait de situer sur des pôles généralement opposés en épistémologie, les différents courants en fonction de conceptions épistémiques auxquelles ils conduisent.

\section{REGARDS SUR LE BESOIN DE POURSUIVRE LA RECHERCHE CONCEPTUELLE}

Considérant ce qui précède, nous voyons qu'il y a de l'espace pour la réflexion et qu'il pourrait être précieux de mener des recherches conceptuelles afin d'organiser de manière plus intelligible l'ensemble de la constellation des différentes notions qui constituent le champ de l'épistémologie en éducation. En ce sens, nous avons proposé un premier essai de classification des rapports aux savoirs par une organisation structurée autour de quatre types de rapports: épistémologique, épistémique, gnoséologique, idiosyncratique et didactique. Essentiellement, cette organisation vise à proposer une distinction entre les divers rapports auxquels les écrits font références, des rapports qui, nous semble-t-il, ne sont pas mutuellement exclusifs malgré qu'ils portent sur des objets différents. Par la suite, nous avons proposé un modèle en axes croisés à partir desquels il serait possible, selon une logique que nous pourrions qualifier de «bipolaire», de situer les différents courants en fonction de mégacritères épistémologiques. Par ailleurs, le caractère foncièrement émergent de ces considérations témoigne du niveau embryonnaire, mais non moins essentiel, d'une réflexion qui va dans le sens d'une synthèse visant à structurer l'information. La poursuite d'un tel exercice permettrait aux chercheurs et enseignants d'œuvrer à l'aide de repères structurés et intelligibles sur lesquels appuyer tant leurs réflexions en matière d'épistémologie en éducation que leurs interventions auprès des élèves. Il y aurait donc, en cette matière, un réel besoin de recherches conceptuelles, un besoin qui ne répondrait par uniquement à des exigences purement conceptuelles, mais également et surtout didactique, voire éthique compte tenus des relations dessinées entre ces rapports, l'enseignement dispensé, les modes d'évaluation employés, la persévérance de même que la réussite scolaire. 
En ce sens, nous croyons que la question des rapports aux savoirs des élèves et des enseignants ne doit pas être considérée comme un luxe ou, pour emprunter les mots de Paul au sujet de la pensée critique, comme un vernis ajouté pour faire joli. Au contraire, ces questions sont fondamentales à l'intérieur de toute dynamique d'enseignement-apprentissage et devraient être considérées comme centrales tant dans la formation initiale que dans l'action enseignante. Il en va en quelque sorte de la réflexivité dans laquelle seront engagés les enseignants face à leur pratique, une réflexivité qui a des conséquences sur le caractère lucide et ludique de leurs interventions. Autant d'éléments donc qui conduisent à penser que la cognition épistémique devrait faire partie intégrante de l'action enseignante et s'articuler non pas au détriment des processus d'appropriation des contenus standardisés, mais avec eux.

\section{NOTES}

1 À l'intérieur de ce texte, le masculin est utilisé à titre épicène.

2 Cette liste a été obtenue par un relevé des notions épistémiques identifiées à l'analyse des différents modèles cités précédemment, d'écrits considérés fondamentaux dans l'épistémologie contemporaine (Kuhn, Popper) ainsi que des postures identifiées dans le cadre d'une recherche antérieure (Gagnon, 2008). 


\section{BIBLIOGRAPHIE}

Bader, B. (2001). Étude des conversations estudiantines autour d'une controverse entre scientifiques sur la question du réchauffement climatique. Thèse de doctorat Québec: Université Laval.

Baxter Magolda, M. B. (2002). Epistemological reflection: The evolution of epistemological assumptions from age 18 to 30. In B. K. Hofer \& P. R. Pintrich (Eds.), Personal epistemology: The psychology of beliefs about knowledge and knowing (pp. 89-102). Mahwah, NJ: Lawrence.

Beillerot, J. (1999). Savoirs. . In J. Houssaye (Ed.), Questions pédagogiques. Encyclopédie historique (pp. 520-531). Paris: Hachette.

Belenky, M. F., Clinchy, B., Goldberger, N. et Tarule, J. M. (1986). Women 's ways of knowing. The development ofself, voice, and mind. New York: Basic Books Inc. Publishers.

Buehl, M., \& Alexander, P. (2002). Beliefs about Schooled Knowledge: Domain Specific or Domain General? Contemporary Educational Psychology 27, 415- 449

Caillot, M. (2001). Rapports aux savoirs et didactiques des sciences. In P. Jonnaert \& S. Laurin (Eds.), Les didactiques des disciplines. Un débat contemporain. Québec: Presses de l'Université du Québec.

Charlot, B. (1997). Du rapport au savoir: éléments pour une théorie. Paris: Anthropos.

Charlot, B. (2003). La problématique du rapport au savoir. In S. Maury \& M Caillot (Eds.), Rapport au savoir et didactiques (pp. 33-50). Paris: Fabert.

Charlot, B., Bautier, E., \& Rochex, J.-Y. (1992). École et savoir dans les banlieues... et ailleurs. Paris: Armand Colin.

Daniel, M.-F., Splitter, L., Slade, C., Lafortune, L., Pallascio, R., \& Mongeau, P. (2004). Dialogical critical thinking: Elements of definitions emerging in the analysis of transcripts from pupils aged 10 to 12 years. Australian journal of education, 48(3), 295-313.

Désautels, J. et Larochelle, M. (1994). Le constructivisme en action: des étudiant et des étudiantes se penchent sur leur idée de science. Revue des sciences de l'éducation, $X X(1), 157-174$

Désautels, J., \& Larochelle, M. (1989). Qu'est-ce que le savoir scientifique? Point de vue d'adolescents et d'adolescentes. Québec: Presses de l'Université Laval.

Dweck, C. S., \& Leggett, E. L. (1988). A social-cognitive approach to motivation personality. Psychological Review, 95, 256-273.
Edman, L., Bart, W., et Robey, J. (2000). The Minnesota test of critical thinking: development, analysis and critical issues. Paper presented at the Annual Meeting of the American Psychological Association.

Edman, L., Robey, J., \& Bart, W. (2002). Critical thinking, belief biais, epistemological assomptions, and the Minnesota test of critical thinking. Paper presented at the Annual Meeting of the American Educational Research Association.

Fourez, G. (2002). La construction des sciences. Les logiques des inventions scientifiques (4e édition). Bruxelles: De Boeck Université.

Gagnon, M. (2008). Étude sur la transversalité de la pensée critique comme compétence : entre «science et technologie», histoire et philosophie au secondaire. Thèse de doctorat. Québec : Université Laval.

Gagnon, M. (accepté). «Examen des possibles relations entre les rapports aux savoirs et la construction d'une pensée critique chez les adolescents». Revue canadienne d'éducation.

Hofer, B. (2000). Dimensionality and Disciplinary Differences in Personal Epistemology. Contemporary Educational Psychology, 25, 378 - 405.

Hofer, B. K. et Pintrich, P. R. (1997). The development of epistemological theories: Beliefs about knowledge and knowing and their relation to learning. Review of educational research, 67(1), 1-34.

Jehng, J.-C. J., Johnson, S. D., and Anderson, R. C. (1993). Schooling and students' epistemo- logical beliefs about learning. Contemp. Educ. Psychol. 18: 23-25.

Jonnaert, P. et Lenoir, Y. (Dir.). Sens des didactiques et didactique du sens. Sherbrooke: Éditions du CRP.

Jonnaert, P. et Vander Borght (1999). Créer des conditions d'apprentissage. Un cadre de référence pour la formation didactique des enseignants. De Boeck : Bruxelles.

King, P. M., \& Kitchener, K. S. (2002). The Reflection Judgment Model: Twenty years of research on epistemic cognition. In B. K. Hofer \& P. R. Pintrich (Eds.), Personal epistemology: The psychology of beliefs about knowledge and knowing (pp. 37-62). Mahwah, NJ: Lawrence Erlbaum.

King, P., et Kitchener, K. S. (1994). Developing reflective judgment. San Francisco: Jossey-Bass.

Kitchener, K. S. (1983). Cognition, Metacognition and Epistemic cognition: A threelevel model of cognitive processing. Hum. Dev., 26(4), 222-232.

Kuhn, D. (1991). The skills of argument. Cambridge, England: Cambridge University Press. 
Kuhn, D. (1999). A developmental model of crtitical thinking. Eduactional Researcher, 28(2), 26 - 46.

Lafortune, L., Deaudelin, C., Doudin, P.-A., \& Martin, D. (2003). Conceptions, croyances et représentations en maths, sciences, et technos. Québec: PUQ.

Larochelle, M., \& Désautels, J. (2004). Descriptions estudiantines de la nature et de la fabrication des savoirs scientifiques. In L. Lafortune, C. Deaudelin, P.-A. Doudin \& D. Martin (Eds.), Conceptions, croyances et représentations en maths, sciences, et technos. Quebec: Presses de l'université du Québec.

Lodewyk, K. (2007). Relations among epistemological beliefs, academic achievement, and task performance in secondary school students. Educational psychology 27(3), 307-327.

Mason, L., \& Boscolo, P. (2004). Role of epistemological understanding and interest in interpreting a controversy and in topic-specific belief change. Contemporary Educational Psychology, 29, 103-128.

Mathy, P. (1997). Histoire des sciences et enseignement des sciences. Quoi ? Pourquoi ? Comment? Revue des questions scientifiques, Idéologies, construction et libérations, 168(4)

Maury, S., \& Caillot, M. (2003). Rapport au savoir et didactiques. Paris: Fabert.

Mosconi, N., Beillerot, 1. et Blanchard-Laville, C. (2000). Formes et formations $d u$ rapport au savoir. Paris: L'Harmattan.

Mujawamariya, D. (2000). De la nature du savoir scientifique à l'enseignement des sciences: l'urgence d'une approche constructiviste dans la formation des enseignants de sciences. Education et francophonie, 28(2), 1-11.

Nietzsche, F. (1873 / 2002). Vérité et mensonge au sens extra-moral. Paris : Babel.

Paulsen, M., \& Wells, C. (1998). Domain differences in the epistemological beliefs of college students. Research in higher education, 39(4), 365-384.

Perry, W. G. (1970). Forms of Intellectual and Ethical Development in the College Years: A scheme. New York: Academic Press.

Popper, K. (1973)(1 $1^{\text {èr }}$ édition 1934). Logique de la découverte scientifique. Paris: Payot.

Qian, G., \& Alvermann, D. (1995). Role of epistemological beliefs and learned helplessness in secondary school students' learning science concepts from text. Journal of educational psychology, 87(2), 282-292.

Schommer-Aikins, M., Duell, K., \& Barker, S. (2003). Epistemological beliefs across domains using Biglan's classification of academic disciplines. Research in Higher Education, 44(3), 347 - 366
Schommer-Aikins, M., Duell, O. K, and Barker, S. A. (April 2001). Domain generality of epistemological beliefs: It's a matter of degree. Paper presented at the American Educational Research Association, Seattle.

Schommer, M. (1993a). Comparisons of beliefs about the nature of knowledge and learning among postsecondary students. Research in higher education, 34(3), 355370

Schommer, M. (1993b). Epistemological development and academic performance among secondary students. Journal of educational psychology, 85(3), 406-411.

Schommer, M. (1994). A emerging conceptualization of epistemological beliefs and their role in learning. In R. Garner \& P. A. Alexander (Eds.), Beliefs about text and instruction with text. Hillsdale, New Jersey: Erlbaum.

Schommer, M., \& Dunnell, P. A. (1997). Epistemological beliefs of gifted high school students. Roeper Review, 19, 153-156.

Schommer, M., \& Easter, M. (2008). Epistemological beliefs' contributions to study strategies of asian americans and european americans. Journal of educational psychology, 100(4), 920-929.

Schommer, M., \& Walker, K. (1995). Are epistemological beliefs similar across domains? . Journal of Educational Psychology, 87, 424-432.

Schommer, M., \& Walker, K. (1997). Epistemological beliefs and valuing school: Considerations for college admissions and retention. Ressources for Higher Education, 38, 173-186.

Schommer, M., Calvert, C., Gariglietti, G., \& Bajaj, A. (1997). The development of epistemological beliefs among secondary students: A longitudinal study Journal of Educational Psychology, 89, 37-40.

Therriault, G. (2008). Postures épistémologiques que développent des étudiants des profils sciences et technologies et univers social au cours de leur formation initiale à l'enseignement secondaire: une analyse de leurs croyances et de leurs rapports aux savoirs. Thèse de doctorat. Rimouski: UQAR.

Venturini, P. (2007). L'envie d'apprendre les sciences. Motivation, attitudes, rapport aux savoirs scientifiques. Paris: Faubert.

Venturini, P., \& Albe, V. (2002). Interprétation des similitudes et différences dans la maîtrise conceptuelle d'étudiants en électromagnétisme à partir de leur(s) rapport(s) au(x) savoir(s). Aster, 35 165-188.

Vygotski, L. (1985). Pensée et langage. Paris: Terrains. 